\title{
Uma Revisão Sistemática sobre Descrição Semântica na Internet das Coisas
}

\section{Title: A Systematic Review about Semantic Description on the Internet of Things}

\author{
Henrique Gurgacz de Almeida ${ }^{1}$, Sean Siqueira ${ }^{1}$ \\ ${ }^{1}$ Programa de Pós-Graduação em Informática - Universidade Federal do Estado do Rio \\ de Janeiro (UNIRIO) \\ Rio de Janeiro, Rio de Janeiro - Brasil \\ \{henrique.gurgacz, sean\}@uniriotec.br
}

\begin{abstract}
The Internet of Things (IOT) is creating an ecosystem of objects connected on Internet. However, these objects are heterogeneous in terms of resources, characteristics, nature, protocols, actions and technologies. In this scenario, the Thing Description (TD) presents itself as an alternative to describe the IOT objects in a standard way and provide, among other possibilities, a better interoperability. Considering the rising research interest and the application opportunities of the TD, this paper presents a systematic review of the theme, aiming to identify applications, techniques, technologies and results using TD. 324 papers were identified and 46 were selected to be analyzed, which were classified in seven different groups of applications.
\end{abstract}

Keywords. Internet of Things; IOT; Things Description; Semantic Description.

Resumo. A Internet das Coisas (Internet of Things - IOT) está criando um ecossistema de objetos conectados à Internet, porém que são heterogêneos em termos de recursos, características, natureza, protocolos, ações e tecnologias. Neste cenário, a descrição das coisas (Thing Description - TD) mostra-se como uma alternativa para descrever de forma padronizada os objetos da IOT e permitir, entre outras possibilidades, uma melhor interoperabilidade. Visto o crescente interesse de pesquisa e as oportunidades de aplicação da TD, este trabalho apresenta uma revisão sistemática deste tema, visando identificar áreas de aplicação, técnicas, tecnologias e resultados com o uso da descrição das coisas. 324 trabalhos foram identificados, dos quais 46 foram selecionados para análise, os quais foram classificados em seis diferentes áreas.

Palavras-Chave. Internet das Coisas; IOT; Descrição das Coisas; Descrição Semântica das Coisas. 


\section{Introdução}

A expansão da Internet das Coisas (Internet of Things - IOT) tem conectado diversos dispositivos, sensores, controladores e softwares na Internet. Cada um deles possui características específicas, provê ou consome dados e executa funções diversas [Barnaghi et al. 2012]. Com tal expansão, a integração entre os dispositivos da IOT e também com sistemas de informação é cada vez mais difícil. Isso se deve principalmente à heterogeneidade desta rede, visto a gama de protocolos de comunicação e de tecnologias embarcadas nas coisas [Huang e Wu 2016], [Mathew et al. 2011], [Jara et al. 2014].

De acordo com Kabisch e Anicid (2016), os grandes desafios da IOT na atualidade estão associados a silos de dados, interoperabilidade entre as coisas, descoberta automática de recursos e dispositivos, além da interpretação precisa de dados da IOT. Gupta e Gupta (2016) citam ainda como desafios da IOT: armazenamento e recuperação de dados, independência humana, privacidade e segurança e conectividade. O uso de semântica na IOT apresenta-se como uma alternativa para estes desafios, podendo ser aplicada para ([Jara et al. 2014], [Kabisch e Anicid 2016], [Manate e Fortis 2014]):

- assegurar que os dados da IOT possam ser compreendidos pelo homem, pelo computador ou pelos sistemas, indiferente da heterogeneidade ou do alto índice de abstração das fontes dos dados;

- gerar dados entendíveis por outros computadores ou humanos, além de permitir a interpretação e a geração de conhecimento;

- tratar do significado preciso dos dados e das propriedades das coisas da IOT;

- permitir a descrição exata da capacidade destes objetos e entender suas características, bem como os dados ou as ações produzidas ou consumidas e as suas interligações; e

- integrar dados e conhecimento da IOT em múltiplos domínios e aplicações.

O uso das tecnologias e dos conceitos semânticos permite integrar os objetos na Web com o uso de ontologias, que permite classificar as várias propriedades e características das coisas [Mathew et al. 2011]. Desta forma, iniciaram-se pesquisas referentes à descrição das coisas (Thing Description - TD), que consiste em utilizar os conceitos de Web Semântica para descrever características dos objetos da IOT e permitir melhor interoperabilidade entre humanos, computadores, coisas e sistemas. Assim, em TD, usam-se metadados, ontologias, Resource Description Framework (RFD) e eXtensible Markup Language (XML), como na Web Semântica. Muitos desses conceitos e suas bases teóricas têm sido utilizados e testados com IOT.

Visto que TD é um tema novo, com grande interesse de pesquisa e que não foram identificadas revisões neste tema, este trabalho consiste na realização de uma revisão sistemática em bases científicas visando identificar soluções, aplicações e resultados do uso de descrição das coisas na IOT, permitindo assim auxiliar novos desenvolvimentos nessa linha de pesquisa. A revisão sistemática restringe-se ao tema da descrição das coisas na IOT, mais especificamente que fazem uso dos conceitos semânticos.

O trabalho está organizado em quatro principais seções. A primeira apresenta a contextualização sobre o tema de $\mathrm{TD}$, a seção a seguir apresenta a metodologia de 
pesquisa aplicada nesta revisão sistemática, as seções seguintes tratam dos resultados e das conclusões.

\section{Contextualização}

A base da descrição das coisas está associada aos conceitos semânticos, por este motivo alguns autores intitularam a descrição das coisas como descrição semântica das coisas [Chun et al. 2015], [Datta e Bonnet 2016]. A semântica, entre outros objetivos, busca a interoperabilidade entre os diferentes atores no meio computacional, no caso da IOT a proposta é a mesma. Conceitualmente, a interoperabilidade semântica na IOT permite que os dispositivos eletrônicos troquem dados entre sistemas, usuários e outros dispositivos. Os dados semanticamente descritos podem ser lidos pelas máquinas e pelos sistemas, o que habilitaria a comunicação e interação autônoma [Barnaghi et al. 2012], [Datta e Bonnet 2016]. Desta forma, a descrição das coisas pode ser utilizada por dispositivos, sistemas e serviços para descrever suas características de modo que outros atores computacionais possam interagir e interpretar dados.

Adicionalmente, ao analisar a IOT, é possível perceber um ambiente extremamente heterogêneo, visto que fabricantes entregam padrões, protocolos, frameworks e formas de acessos distintas nos seus dispositivos. Isso torna a comunicação e interoperabilidade um grande desafio para a IOT [Borgia 2014]. Com isso, a adoção da descrição das coisas no ambiente da IOT mostra-se como uma possível ferramenta para tratar os desafios da área.

Apesar de ser um tema recente de pesquisa, há uma série de trabalhos que apresentam formas distintas de descrever as características dos objetos da IOT, a principal delas, foi apresentada em 2015 pelo o grupo de pesquisas Web of Things do consórcio W3C. O consórcio propôs uma força tarefa sobre pesquisas referentes à descrição das coisas [W3C 2015]. Os objetivos do grupo são:

- esclarecer e definir como TD pode ser utilizado;

- esclarecer e definir quais aspectos dos dispositivos da IOT podem ser descritos;

- definir um modelo de dados para TD;

- definir um vocabulário para TD; e

- definir um formato e sintaxe adequados para TD.

Em TD, cada ação, propriedade, evento e demais características dos dispositivos da IOT podem ser descritos usando vocabulários padronizados. Na figura 1 pode-se visualizar um exemplo de descrição de um dispositivo do tipo termômetro, onde há uma saída de dados do tipo numérico chamado temperatura, que é acessível por meio de uma URI. A figura 1 apresenta a descrição baseada no padrão em desenvolvimento pela W3C. 


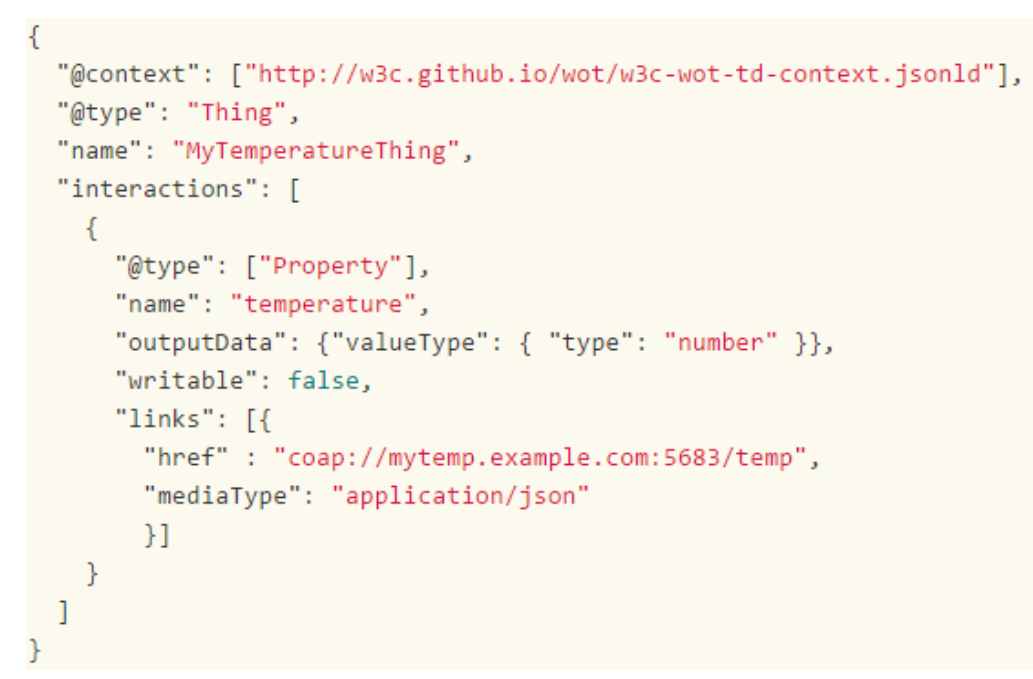

Figura 1: Exemplo de uma descrição de um dispositivo [W3C 2015]

\section{Metodologia}

Para execução desta revisão sistemática tomou-se como base as "Diretrizes para Realização de Revisões de Literatura Sistemática em Engenharia de Software" [Kitchenham e Charters 2007], que recomenda a execução dos seguintes passos:

1 - Definição das questões de pesquisa;

2 - Definição dos parâmetros de busca nas bases científicas;

3 - Definição dos critérios de inclusão e descarte no processo de seleção dos trabalhos;

4 - Seleção dos trabalhos;

5 - Extração dos trabalhos; e

6 - Extração dos resultados.

Desta forma, de acordo com o tema e os objetivos já mencionados, foram elaboradas duas questões de pesquisa para esta revisão sistemática, são elas:

- Como Thing Description tem sido utilizada com a IOT?

- Quais os resultados estão sendo observados com o uso de Thing Description na IOT?

Para execução das buscas por artigos foram escolhidas no portal da Capes ${ }^{1}$ as cinco maiores bases na área de ciência da computação, conforme classificação da Capes. As bases selecionadas foram: ACM, IEEE, Scopus, Science Direct e Web of Science. Em todas as bases, os parâmetros de busca foram aplicados apenas para o título, palavras-chave e resumo. Não foram aplicados filtros por ano, por área de conhecimento ou quaisquer atributos das bases científicas. Na tabela 1 é possível visualizar o parâmetro de busca utilizado nas respectivas bases utilizadas.

\footnotetext{
${ }^{1}$ Portal da Capes: Portal de Periódicos que tem como missão promover o fortalecimento dos programas de pós-graduação no Brasil por meio da democratização do acesso online à informação científica internacional de alto nível [Capes 2016]. 
Tabela 1: Parâmetros de busca

\begin{tabular}{|l|l|}
\hline Base & Parâmetros \\
\hline ACM e IEEE & $\begin{array}{l}\text { ((iot OR "Internet of Things" OR wot OR "Web of Things" OR "Internet of } \\
\text { Everything") AND ("semantic descr*" OR "things descr" OR "thing } \\
\text { descr*" OR "semantic schema" OR "semantic model*" OR "description of } \\
\text { things" OR "service descr*")) }\end{array}$ \\
\hline Science Direct & $\begin{array}{l}\text { tak(((iot OR "Internet of Things" OR wot OR "Web of Things" OR "Internet } \\
\text { of Everything") AND ("semantic descr*" OR "things descr*" OR "thing } \\
\text { descr*" OR "semantic schema" OR "semantic model*" OR "description of } \\
\text { things" OR "service descr*"))) }\end{array}$ \\
\hline Web of Science & $\begin{array}{l}\text { TS=((iot OR "Intemet of Things" OR wot OR "Web of Things" OR "Internet } \\
\text { of Everything") AND ("semantic descr*" OR "things descr*" OR "thing } \\
\text { descr*" OR "semantic schema" OR "semantic model } \\
\text { things" OR "description of }\end{array}$ \\
\hline Scopus & $\begin{array}{l}\text { TITLE-ABS-KEY((iot OR "Internet of Things" OR wot OR "Web of } \\
\text { Things" OR "Internet of Everything") AND ("semantic descr*" OR "things } \\
\text { descr*" OR "thing descr*" OR "semantic schema" OR "semantic model*" } \\
\text { OR "description of things" OR "service descr*")) }\end{array}$ \\
\hline
\end{tabular}

Os termos utilizados para a busca foram:

- Internet Of Things: refere-se ao termo principal de busca para esta revisão sistemática;

- Web Of Things: refere-se a uma especialização da IOT em que o protocolo Web é utilizado para conectar as coisas [Jara et al. 2014];

- IOT e WOT: referem-se às siglas para Internet Of Things e Web Of Things respectivamente;

- Internet of Everything: conceito criado pela empresa multinacional Cisco em 2012 como uma extensão da IOT. A Internet of Everything abrange pessoas, processos, dados e coisas de forma inteligente [Cisco 2017];

- Semantic descr*: termo utilizado para encontrar artigos relacionados à descrição semântica;

- Things descr* e Thing descr*: termos utilizados para encontrar artigos relacionados à descrição das coisas;

- Semantic schema: refere-se à busca por esquemas semânticos;

- $\quad$ Semantic model*: refere-se à busca por modelos e modelagens semânticos, que podem ser aplicados usando descrição das coisas;

- $\quad$ Description of things: Termo utilizado para buscar por descrição das coisas; e

- $\quad$ Service descr*: termo utilizado para a busca por descrição de serviços.

A execução da busca e a extração dos dados foram executadas em 02/01/2018 e tiveram como resultado 324 artigos. Na figura 2 pode ser observada a quantidade de artigos por base científica, considerando o total de artigos. 


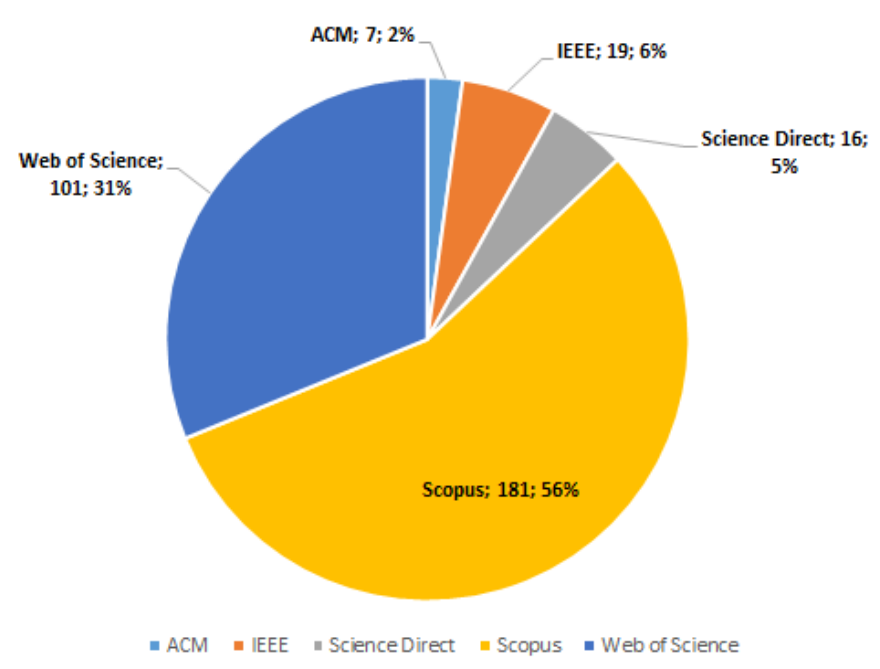

Figura 2: Quantidade de artigos por base científica

Para auxiliar na validação da efetividade dos parâmetros de busca aplicados, três artigos de referência ([Mathew 2011], [Christophe 2011], [Datta e Bonnet 2016]), já conhecidos pelos autores, foram selecionados e deveriam constar nos resultados das buscas. Conforme esperado, estes três artigos foram encontrados nos resultados da busca nas bases científicas e, desta forma, foi possível passar para a próxima fase do processo de revisão sistemática.

$\mathrm{Na}$ fase de seleção, foram identificados os artigos duplicados e que estavam fora do escopo da pesquisa, com base na análise do título e do resumo. Ambos foram descartados da lista de artigos selecionados. Na figura 3 é possível visualizar que entre os 324 artigos extraídos das bases científicas, 110 estavam duplicados e 90 estavam fora do escopo. Os critérios de descarte por escopo (destes 44 artigos) podem ser vistos na figura 4. O principal motivo foi por não tratar de descrição das coisas ou de IOT.

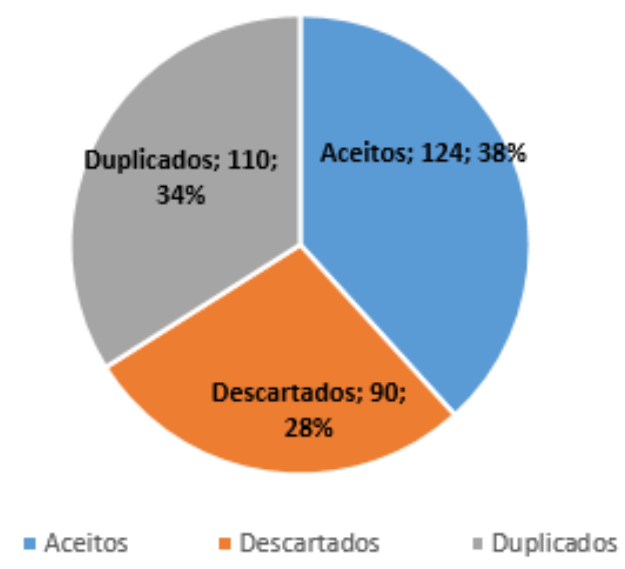

Figura 3: Resultado da fase de seleção 


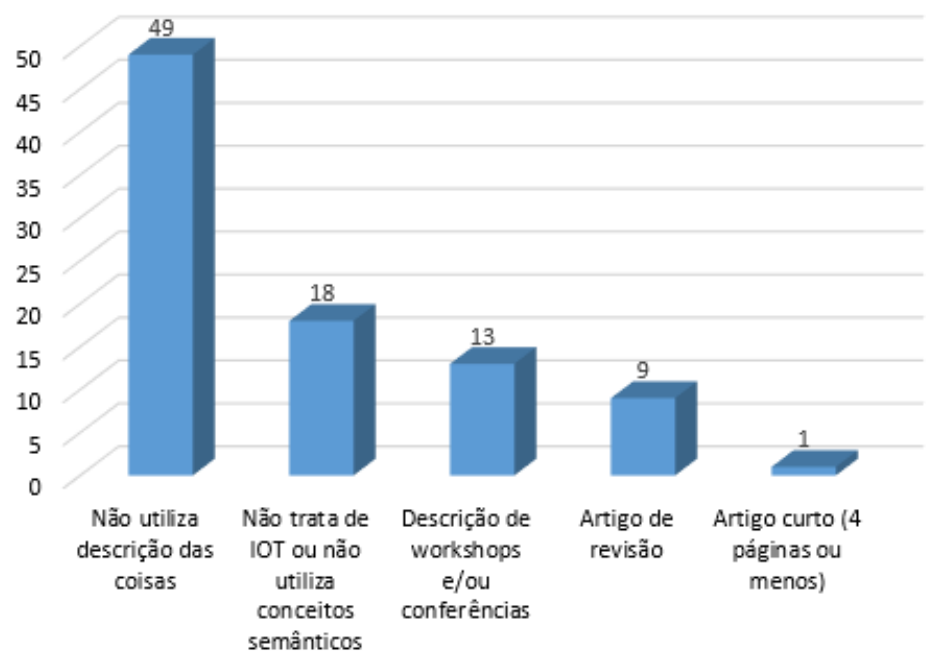

Figura 4: Motivo de descarte na fase de seleção

Com a fase de seleção concluída, foram extraídos de forma completa todos os 124 trabalhos selecionados. Durante a fase de análise dos artigos completos também houve artigos duplicados e descartados. Na figura 5 é possível visualizar o resultado após a fase de extração e análise, em que dos 124 artigos analisados 46 foram aceitos.

Na figura 6 é possível visualizar os motivos de descarte dos trabalhos nesta fase. Foram identificados três principais motivos de descarte nesta fase. O primeiro refere-se a trabalhos que não utilizaram descrição das coisas. O segundo motivo de descarte envolve os trabalhos que não tratam de IOT ou que não usaram conceitos semânticos. Por fim, o terceiro motivo de descarte envolvem artigos curtos, com quatro páginas ou menos. Estes, na maioria, não trazem detalhes suficientes para fazer uma avaliação dos resultados, métodos ou formas de aplicação. Os artigos válidos tiveram as características de aplicação, tecnologias e resultados anotadas à partir de uma leitura detalhada dos artigos completos.

Os trabalhos classificados como "Trabalho não disponibilizado", são aqueles que não estavam disponíveis para download nas bases científicas e que mesmo após tentativas de contato com os autores não houve sucesso ou resposta para obtenção dos trabalhos.

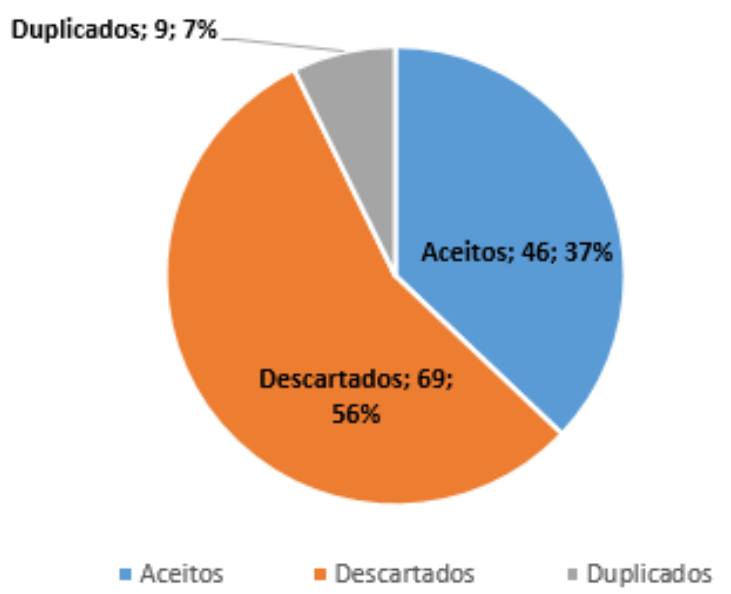

Figura 5: Resultado da fase de extração 


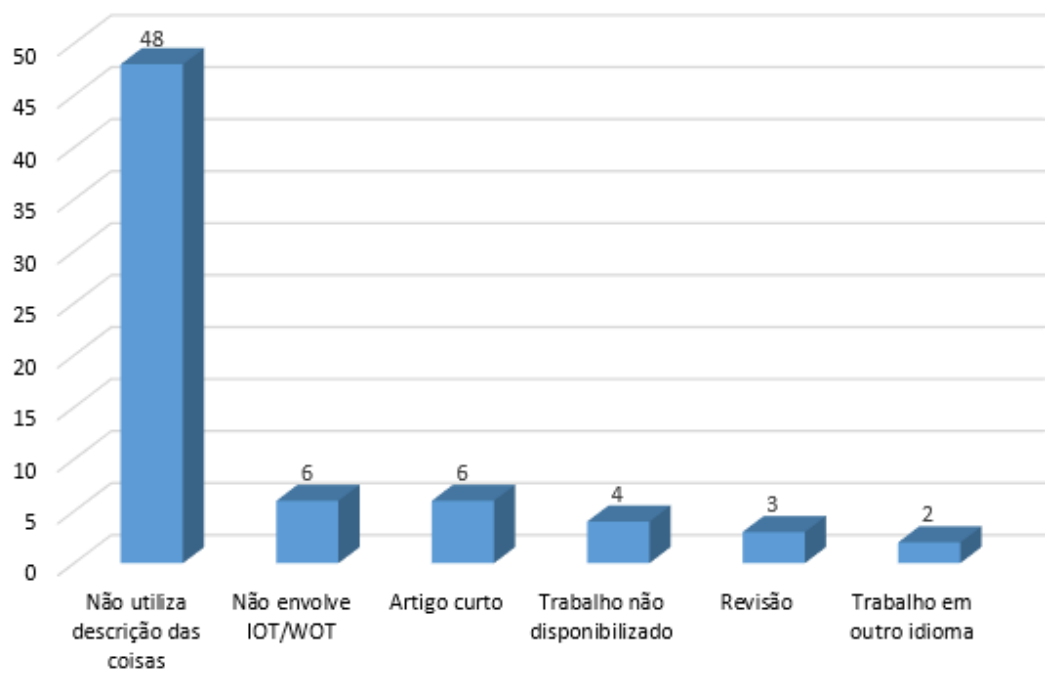

Figura 6: Motivo de descarte de trabalhos na fase de extração

$\mathrm{Na}$ seção de apêndices deste trabalho foi disponibilizada uma tabela com os dados de todos os artigos selecionados nesta revisão sistemática.

Para execução de todos os procedimentos citados nesta seção foram utilizadas as seguintes ferramentas:

- $\quad$ Start $^{2}$ : Software disponibilizado pela Universidade Federal de São Carlos para as fases de seleção e extração;

- $\quad$ Mendeley $^{3}$ : Software utilizado como repositório dos trabalhos extraídos e também utilizado para anotação e citação; e

- $\quad$ Microsoft Excel: Software utilizado para geração de gráficos, análise de dados e anotações.

\section{Resultados}

Cada um dos 46 artigos selecionados na fase de extração foi categorizado quanto a aplicação, técnica/tecnologia e resultados. Na figura 7 é possível visualizar as aplicações de TD identificadas, são elas:

- Comunicação na IOT: trabalhos que utilizam descrição das coisas para uma melhor comunicação e interoperabilidade no ambiente da IOT;

- Comunicação Máquina-Máquina: Trabalhos que utilizam a descrição das coisas para possibilitar que dispositivos da IOT comuniquem-se de forma autônoma;

- Busca e Descoberta de Serviços ou Coisas: trabalhos que se preocupam em buscar ou descobrir coisas semanticamente descritas;

- Privacidade, Transparência ou Segurança: trabalhos que utilizam a descrição das coisas para melhorar critérios de segurança, transparência ou segurança do ambiente da IOT;

\footnotetext{
${ }^{2}$ Start: http://lapes.dc.ufscar.br/tools/start_tool

${ }^{3}$ Mendeley: https://www.mendeley.com 
- Otimização de Recursos ou Desempenho: trabalhos que se preocuparam com desempenho ou otimização no processo de comunicação, processamento, interpretação ou busca;

- Outros: trabalhos que tratam de outros assuntos diversos, tais como padrões, processos e provisionamento de objetos da IOT.

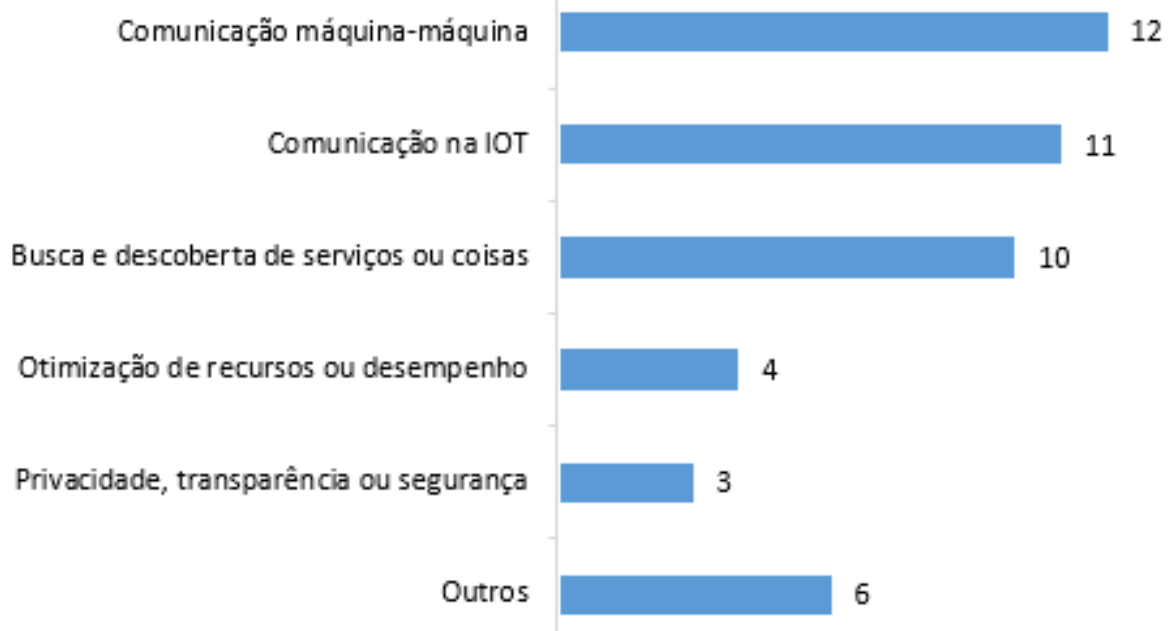

Figura 7: Principais aplicações de TD identificadas

Além das principais aplicações de TD, foram identificadas também as principais técnicas e tecnologias utilizadas nos trabalhos. Neste contexto, foram mapeadas as seguintes técnicas e tecnologias:

- Ontologias diversas;

- Arquitetura orientada a serviços (SOA);

- Tecnologias semânticas (RDF, OWL e SPARQL);

- Ontologia de rede semântica de sensores (Semantic Sensor Network Ontology SSN);

- Linguagem de marcação de sensores (Sensor Markup Language - SENML);

- Dados/Serviços conectados;

- Notação de objetos JavaScript (JavaScript Object Notation - JSON);

Para critério de avaliação e comparação, apenas as técnicas ou tecnologias mais utilizadas em TD foram listadas na tabela 2:

Tabela 2: Técnicas e tecnologias utilizadas em TD

\begin{tabular}{|l|r|}
\hline Tecnologia & Quantidade \\
\hline Ontologias diversas & 14 \\
\hline SOA & 10 \\
\hline RDF, OWL e SPARQL & 9 \\
\hline Semantic Sensor Network Ontology (SSN) & 8 \\
\hline XML e Sensor Markup Language & 8 \\
\hline Dados ou Serviços Conectados & 3 \\
\hline JSON & 3 \\
\hline
\end{tabular}

Alguns trabalhos utilizam mais de uma técnica/tecnologia em TD, por este motivo a somatória da quantidade é superior ao número de trabalhos selecionados. 
Visto que TD tem como principal base os conceitos da web semântica [Datta e Bonnet 2016], o alto uso de ontologias, RDF, OWL, SPARQL e da ontologia SSN era esperado. Uma importante descoberta que pode ser percebida na tabela 2 é o considerável uso de SOA bem como linguagens de marcação XML, como por exemplo a Sensor Markup Language (SENML).

As seções a seguir apresentam as aplicações de TD identificadas nos trabalhos encontrados. Nestas seções serão citados os principais trabalhos, suas técnicas e tecnologias.

\subsection{Comunicação na IOT e Comunicação Máquina-Máquina (M2M)}

As categorias de aplicação de TD que tratam da comunicação (Comunicação na IOT e Comunicação Máquina-Máquina) representam metade de todos os trabalhos analisados.

Datta e Bonnet (2016) demonstraram o uso de JavaScript Object Notation for Linked Data (JSON-LD) para descrever atributos e propriedades dos objetos da IOT e com isso conseguiram contribuir positivamente para a construção de um framework com o objetivo maior de permitir a comunicação M2M. Foram encontrados também outros dois trabalhos similares ([Yachir et al. 2016] e [Huang e Wu 2016]) que utilizam a mesma tecnologia, onde foram descritos não apenas os atributos e propriedades dos objetos, mas também eventos, resultados, ações e interligação entre as coisas.

Referente à comunicação M2M, Bovet e Hennebert (2014) apresentaram um framework para comunicação $\mathrm{M} 2 \mathrm{M}$ aplicado à eficiência energética em edifícios inteligentes (Smart Buildings). Neste trabalho foram utilizadas ontologias existentes e novas. Foram utilizados SOA, descoberta de serviços, RDF e SPARQL, entre outras tecnologias. Apesar da limitação da comunicação M2M apenas entre alguns dispositivos específicos, esse trabalho obteve êxito ao conseguir interação entre os objetos da IOT de forma autônoma.

Sozinha, a ontologia SSN, utilizada em diversos trabalhos, não consegue representar o rico modelo de informações da infraestrutura da IOT, bem como suas interligações e dados [Ali et al. 2015]. Neste cenário, é necessária a criação de novas ontologias para os diversos domínios da IOT. Tal necessidade ficou perceptível pela grande gama de trabalhos que criaram ontologias novas, como: [Kotis et al. 2012], [De 2011], [Bovet e Hennebert 2014], [Sulistyo e Prinz 2011], [Fredj et al. 2013] e [Alam e Noll 2010].

O primeiro trabalho a utilizar os padrões de TD, que estão sendo estudados pela W3C [W3C 2015], foi Semantic Web Thing Architecture, em que foram realizadas simulações em laboratório com alguns dispositivos eletrônicos utilizando TD baseado nos padrões propostos pela W3C [Mazayev et al. 2017]. Como resultados os autores citaram que alguns padrões ainda precisam ser melhor descritos e testados, como o uso de um comando único partindo de um dispositivo para comunicar-se de forma interativa com vários outros, como no caso de um comando tentar desligar as luzes de vários dispositivos ao mesmo tempo.

Diferente dos demais trabalhos, no estudo de Bao et al. (2012) notou-se o uso de poucos conceitos semânticos para representação de objetos. O padrão XML foi utilizado para descrever as características dos objetos e foram utilizadas redes neurais para identificar serviços na IOT e facilitar assim a comunicação com as coisas. 
Por fim, com o objetivo de permitir a interoperabilidade na IOT foram utilizados dados e serviços conectados. Thoma et al. (2013) propuseram solucionar os desafios da comunicação M2M dirigidas a processos de negócio utilizando SOA, serviços conectados e sem a necessidade de um barramento (Enteprise Service BUS - ESB). A descrição das coisas foi utilizada no formato de descrição dos serviços, sendo que cada serviço representava um objeto da IOT. Por outro lado, em [Yang et al. 2010] o objetivo foi justamente criar um barramento (ESB) para criar serviços que representassem coisas da IOT e conseguir desta forma a comunicação no ambiente heterogêneo da IOT.

Os seguintes trabalhos também foram selecionados por utilizarem TD e Comunicação/M2M, porém não foram detalhados por utilizarem TD com métodos e resultados semelhantes aos já descritos nos trabalhos acima: [Han et al. 2015], [Khriyenko et al. 2012], [Alam e Noll 2010], [Yongan et al. 2016], [Sahlmann e Schwotzer 2015], [Brunner et al. 2009], [Wang et al. 2013], [Cho et al. 2015], [Charpenay et al. 2015].

\subsection{Busca e Descoberta de Serviços ou Coisas}

Apenas disponibilizar novos objetos na internet não é suficiente para criar um ambiente integrado e com comunicação inteligente na IOT. Neste sentido, conseguir buscar ou descobrir os objetos e serviços corretos na internet é fundamental nesse processo. Desta forma, alguns trabalhos conseguiram buscar objetos ou serviços na internet utilizando a descrição das coisas.

Criar uma interface visual e amigável onde o usuário final possa buscar por dados ou ações para serem executados em objetos semanticamente descritos na IOT foi o objetivo de Ara et al. (2014). Neste trabalho foi utilizada a composição de serviços para representar objetos reais da IOT, onde cada serviço armazena as características dos objetos no formato RDF/XML e para a busca foi usada a linguagem SPARQL.

Já no trabalho de Mingozzi et al. (2016) foi implementada uma plataforma com descrição detalhada das coisas. Foi criado também um mecanismo de busca de objetos da IOT de forma que no caso de falha ou má qualidade dos dados de algum dispositivo, a solução garantiria a melhor disponibilidade na comunicação e nos dados transitados, sugerindo outros dispositivos similares. Este trabalho usou a ontologia SSN para descrever as características dos objetos.

Diferente dos trabalhos anteriores, Forestiero (2017) utilizou agentes e um padrão próprio de descrição das coisas no formato XML. O objetivo do trabalho foi efetuar a busca por dispositivos semelhantes. Para isso foi usado um ambiente simulado com centenas de objetos da IOT. A solução proposta conseguiu recomendar dispositivos semelhantes baseado em critérios de busca informados pelo usuário.

Por fim, no trabalho Pervasive Device and Service Discovery Protocol In XBee Sensor Network [Akbar et al. 2017] foi utilizada a ontologia SSN para descrição das coisas e foi criado um protocolo próprio. Foi utilizada a técnica de replicar para todos os dispositivos da rede a descrição semântica de um novo objeto na rede, permitindo assim que todos os dispositivos tivessem a descrição de todos os demais, facilitando o processo de busca dos dispositivos. Este trabalho limitou-se em testar apenas três dispositivos, porém foi apresentado como trabalhos futuros testar em um ambiente maior ou simulado. 
Os seguintes trabalhos também foram identificados com o objetivo de efetuar busca ou descoberta das coisas, porém não foram detalhados por utilizarem os mesmos métodos e chegarem a resultados semelhantes com o uso de TD aos dos trabalhos acima apresentados: [Zhou et al. 2015], [Liu et al. 2016], [Jia et al. 2017], [Mayer e Zurich 2013], [Fredj et al. 2013] e [Gao e Derguech 2013].

\subsection{Otimização de Recursos ou Desempenho}

O uso de TD também foi notado para otimização de recursos e desempenho na IOT em três trabalhos.

No primeiro trabalho, Albalas et al. (2017) fizeram uso de descrição das coisas para objetos da IOT com o objetivo de reduzir o consumo de energia elétrica dos dispositivos. Neste trabalho os dispositivos foram representados por serviços baseados em SOA. Foi usada a série de Fibonacci para reduzir a frequência de requisições feitas diretamente aos consumos conforme a redução de bateria. O resultado fui uma redução de $75 \%$ no consumo e requisições ao dispositivo.

Já no trabalho de Hur et al. (2015) foi feita uma comparação da utilização de plataformas na nuvem para comunicação com objetos da IOT. Foram utilizados como exemplos objetos semanticamente descritos para serem localizados e comunicados a partir de cada plataforma. Neste trabalho foi proposta uma ontologia nova, chamada de $\mathrm{SSD}$, onde foram descritos os objetos utilizados no experimento. O experimento envolveu diferentes tipos de objetos, tais como: Raspberry, Arduino e Smart Phones. A comparação foi feita com três plataformas IOT na nuvem e mostram melhor comportamento com a plataforma comercial Carriots.

No trabalho de Etelapera et al. (2014) foi utilizada TD para descrever os objetos e conseguir com isso maior eficiência energética. Neste trabalho alguns dados referente a objetos da IOT foram armazenados no formato RDF e tais dados foram consultados para identificar o consumo de energia e situação da bateria de cada equipamento. No experimento com estações telemétricas, foi possível reduzir em $47,9 \%$ o consumo de energia usando o algoritmo proposto para compressão do tráfego de dados, apoiado por TD.

Por fim, Cho et al. (2015) usaram TD como ferramenta para melhorar a eficiência na comunicação e compartilhamento de dados entre os objetos da IOT. Neste trabalho foi proposto um proxy que utilizou descrição semântica das coisas no formado SSN. O trabalho conseguiu demonstrar a redução do tráfego de dados na rede dos objetos com o uso do proxy que já possuía a descrição de todos os objetos da rede e assim evitadas chamadas desnecessárias aos objetos.

\subsection{Privacidade, Transparência ou Segurança}

Descrição das coisas também mostrou-se aplicável para a privacidade, transparência e segurança na IOT. Alguns exemplos de aplicações e resultados foram:

A implementação do projeto T3 baseado em metadados e descrição de coisas da IOT foi apresentado nos trabalhos [Pignotti et al. 2014] e [Pignotti e Edwards 2013], em que objetivou-se a descrição de quais dados e interações são realizados entre sistemas e objetos. Foi desenvolvida uma interface para dispositivos móveis onde o usuário poderia escolher se desejava aceitar ou não a comunicação e troca de dados com objetos 
da IOT. O uso de RDF, OWL e ontologias já disponibilizadas foi fundamental para o sucesso do trabalho.

Outro projeto semelhante foi apresentado por Celdran et al. (2016), no qual o foco foi na privacidade em preservar a localização dos usuários na integração com objetos da IOT. Da mesma forma, uma combinação de ontologias foi utilizada para conseguir descrever os objetos, onde eles estavam e o que eles visavam buscar dos usuários finais.

\subsection{Outras Aplicações}

Outras áreas de aplicação também utilizaram TD. Em [Dai e Wang 2010] foi proposta uma extensão da linguagem de marcação WSDL para conseguir descrever não apenas os serviços, mas também as características dos objetos por trás de cada serviço.

$\mathrm{Su}$ et al. (2014) defendem que a linguagem de marcação SenML tem sido amplamente utilizado para armazenar as propriedades de objetos da IOT, porém tal linguagem não possui características semânticas e por este motivo foi proposto um trabalho para realizar a conversão de objetos descritos no formato SenML para o formado RDF. Neste trabalho foram percebidas algumas dificuldades, visto que o formato SenML não consegue gerar uma descrição semântica de objetos da IOT usando todos os recursos do formato RDF.

Já no trabalho de Hirmer et al. (2016) foi proposta uma abordagem baseada em ontologia para registrar e provisionar coisas da IOT. Para este fim foi criada a Plataforma de gerenciamento de recursos (Resource Management Platform - RMP), onde foi necessário acoplar a linguagem de marcação SenML para registrar as características dos objetos da IOT assim que o provisionamento é feito. O trabalho conseguiu reduzir o tempo e complexidade de implantação e provisionamento de objetos da IOT. Características como segurança e privacidade foram colocadas como trabalhos futuros.

O uso de uma descrição formal própria de objetos da IOT para serem utilizados no desenvolvimento de processos de negócio foi aplicado no trabalho [Suri et al. 2017]. Neste trabalho foi criado um framework que permitiu aos usuários desenhar processos de negócio que comunicam-se com objetos da IOT e executam ações orquestradas pelo usuário. Como resultado foi apresentada apenas uma simulação de um processo de negócio e como trabalhos futuros foi proposto implementar processos maiores e mais complexos.

A criação de um novo padrão para descrever coisas em um ambiente residencial ou predial foi proposto por Schachinger et al. (2017), onde foi definido um vocabulário específico para objetos da IOT que tratam de automação residencial e predial.

Por fim, Christophe (2012) apresentou a descrição de lugares e a associação destes lugares com objetos também descritos semanticamente. A união de algumas ontologias foi necessária para este projeto, que objetivou mapear a interação dos objetos da IOT com objetos reais.

\section{Conclusões}

A expansão da IOT traz vários desafios a serem tratados, entre os quais podemos citar a heterogeneidade do ambiente, onde diversos dispositivos com propriedades e 
características diferentes estão em uma mesma rede. Para tratar este desafio e muitos outros, os conceitos da web semântica tem sido aplicados na IOT. Neste cenário, a TD mostra-se como uma importante ferramenta, capaz de auxiliar em diversas questões na IOT.

Para responder a primeira questão de pesquisa desta revisão sistemática, a saber, "Como TD tem sido utilizada com IOT?", precisamos citar as principais aplicações identificadas, são elas: comunicação na IOT, comunicação M2M, busca, privacidade, transparência e segurança. Além disso não podemos deixar de citar as ferramentas e tecnologias que foram percebidas nestas aplicações. São elas: Ontologias, SOA, RDF, OWL, SPARQL, SSN, XML e SenML.

Ainda referente ao uso de TD, é importante ressaltar o uso frequente de comunicação baseada na web, demonstrando assim que muitos trabalhos, mesmo sem citar tal característica, utilizam a web das coisas (Web Of Things - WOT) para comunicação.

Referente a questão de pesquisa sobre os resultados observados com o uso de TD, a efetiva inferência semântica, bem como a comunicação M2M eficaz e inteligente utilizando TD, ainda não podem ser totalmente demonstradas se considerarmos a real heterogeneidade do ambiente da IOT. Além disso é importante ressaltar os bons resultados referentes a busca de dispositivos descritos através da TD. Por fim, percebeuse a falta de padrões e processos no uso de TD, como por exemplo na tentativa de criação de ontologias, ou adaptação de ontologias para dispositivos da IOT. Outro exemplo é a falta de padrão na forma da descrição que cada pesquisa adotou formas diferentes de descrição das coisas.

Como trabalhos futuros podemos citar a necessidade de avaliar se os padrões que estão sendo criados pelo consórcio W3C são compatíveis para as pesquisas aqui levantadas. Este trabalho poderá identificar oportunidades de melhorias nos padrões que estão sendo estabelecidos. A falta de padrões e processos com o uso da TD é recomendado como outro trabalho futuro, no sentido de buscar melhores práticas e processos na aplicação da TD.

\section{Referências}

Akbar, S. R., Kurniawan, W., Ichsan, M. H. H., Arwani, I., e Handono, M. T. (2017). "Pervasive device and service discovery protocol in XBee sensor network". International Conference on Advanced Computer Science and Information Systems ICACSIS. pp. 79-84. [GS Search]

Alam, S., Noll, J. (2010). "A semantic enhanced service proxy framework for internet of things". Proceedings - IEEE/ACM International Conference on Green Computing and Communications - GreenCom 2010, International Conference on Cyber, Physical and Social Computing - CPSCom 2010, pp. 488-495 [ㄹesearch Gate]

Albalas, F., Mardini, W., Al-Soud, M. (2017). “AFT: Adaptive Fibonacci-based Tuning Protocol for Service and Resource discovery in the Internet of Things". The 2nd 
International Conference on Fog and Mobile Edge Computing (FMEC 2017). pp. 177- 182. [Research Gate]

Ali, M. I., Ono, N., Kaysar, M., Griffin, K., Mileo, A. (2015). “A Semantic Processing Framework for IoT-Enabled Communication Systems". International Semantic Web Conference - ISWC 2015. pp. 241-258. [GS Search]

Ara, S. S., Shamszaman, Z. U., and Chong, I. (2014). "Web-of-objects based usercentric semantic service composition methodology in the internet of things". International Journal of Distributed Sensor Networks. [http://journals.sagepub.com/doi/abs/10.1155/2014/482873]

Bao, J., Ding, Y., and Hu, H. (2012). “A new service selection algorithm in USPIOT”. IEEE International Conference on Computer Science and Automation Engineering. [GS Search]

Barnaghi, P., Wang, W., Henson, C., Taylor, K. (2012). "Semantics for the Internet of Things". International Journal on Semantic Web and Information Systems, pp. 1-21.[ https://dl.acm.org/citation.cfm?id=2441528]

Borgia, E. (2014). "The internet of things vision: Key features, applications and open issues". Computer Communications, 54:1-31. [GS Search]

Bovet, G., Hennebert, J. (2014). "Distributed semantic discovery for web-of-things enabled smart buildings". 6th International Conference on New Technologies, Mobility and Security - Proceedings of NTMS 2014 Conference and Workshops. [GS Search]

Brunner, J.-S., Goudou, J.-F., Gatellier, P., Beck, J., Laporte, C.-E. (2009). "SEMbySEM: a framework for sensor management". 1st Int. Workshop on the Semantic Sensor Web (SemSensWeb), collocated with ESWC, pp. 1-15. [Research Gate]

Capes (2016). "Histórico do portal de periódicas da capes". [https://www.periodicos.capes.gov.br/?option $=$ com pcontent\&alias=histórico]. Dezembro-2016.

Celdrán, A. H., Clemente, F. J. G., P'erez, M. G., and P'erez, G. M. (2016). “SeCoMan: A Semantic-Aware Policy Framework for Developing Privacy-Preserving and Context-Aware Smart Applications”. IEEE Systems Journal, pp. 1111-1124. [GS Search]

Charpenay, V., Kabisch, S., Anicic, D., and Kosch, H. (2015). "An ontology design pattern for IoT device tagging systems". Proceedings - 2015 5th International Conference on the Internet of Things, IoT 2015, pages 138-145. [GS Search]

Cho, G., Chun, S., Jin, X., and Lee, K.-H. (2015). "Enhancing CoAP proxy for semantic composition and multicast communication". ACM International Joint Conference on Pervasive and Ubiquitous Computing and ACM International Symposium on Wearable Computers - UbiComp. pp. 7-11.[GS Search]

Christophe, B. (2011). "Semantic profiles to model the Web of Things". Proceedings 7th International Conference on Semantics, Knowledge, and Grids, SKG 2011, pp. 51-58. [GS Search]

iSys: Revista Brasileira de Sistemas de Informação (isys: Brazilian Journal of Information Systems) 
Christophe, B. (2012). "Managing massive data of the internet of things through cooperative semantic nodes". Proceedings - IEEE 6th International Conference on Semantic Computing, ICSC 2012, pp. 93-100. [GS Search]

Ch'un, S., Seo, S., Oh, B., and Lee, K. H. (2015). "Semantic description, discovery and integration for the Internet of Things". Proceedings of the 2015 IEEE 9th International Conference on Semantic Computing, IEEE ICSC 2015. [GS Search]

Cisco (2017). "Internet of everything”, [http://ioeassessment.cisco.com/]. Janeiro-2017.

Dai, C. and Wang, Z. (2010). "A flexible extension of WSDL to describe non-functional attributes". 2nd International Conference on E-Business and Information System Security, EBISS2010. [GS Search]

Datta, S. K. and Bonnet, C. (2016). "Describing things in the Internet of Things: From CoRE link format to semantic based descriptions". IEEE International Conference on Consumer Electronics-Taiwan, ICCE-TW 2016. [http://www.eurecom.fr/en/publication/4848/download/cm-publi-4848.pdf]

De, S., Barnaghi, P., Bauer, M., and Meissner, S. (2011). "Service modelling for the Internet of Things". 2011 Federated Conference on Computer Science and Information Systems (FedCSIS), pp. 949-955. [GS Search]

Etelapera, Matti; Giaffreda, Raffaele; Vecchio, M. (2014). "Improving Energy Efficiency in IoT with Re-configurable Virtual Objects". IEEE World Forum on Internet of Things, pp. 520-525. [GS Search]

Forestiero, A. (2017). "Multi-Agent Recommendation System in Internet of Things". 17th IEEE/ACM International Symposium on Cluster, Cloud and Grid Computing. [GS Search]

Fredj, S. B., Boussard, M., Kofman, D., and Noirie, L. (2013). "A scalable IoT service search based on clustering and aggregation". IEEE International Conference on Green Computing and Communications and IEEE Internet of Things and IEEE Cyber, Physical and Social Computing, GreenCom-iThings-CPSCom 2013, pp. 403410. [GS Search]

Gao, F. and Derguech, W. (2013). "Ubiquitous service capability modeling and similarity based searching". Lecture Notes in Computer Science (including subseries Lecture Notes in Artificial Intelligence and Lecture Notes in Bioinformatics), pp. 173-184. [GS Search]

Gupta, Reetu e Gupta, Rahul. (2016). "ABC of Internet of Things: Advancements, benefits, challenges, enablers and facilities of IoT". 2016 Symposium on Colossal Data Analysis and Networking (CDAN), pp. 1-5. [http://www.academia.edu/download/50183615/Reetu IEEE paper.pdf]

Han, S. N., Lee, G. M., Crespi, N., Luong, N. V., Heo, K., Brut, M., and Gatellier, P. (2015). "DPWSim: A Devices Profile for Web Services". IEEE Internet of Things Journal, pp. 221-229. [http://researchonline.ljmu.ac.uk/288/1/IoT-0250-2014.pdf]

Hirmer, P.,Wieland, M., Breitenb"ucher, U., and Mitschang, B. (2016). "Automated Sensor Registration, Binding and Sensor Data Provisioning". 28th International Conference on Advanced Information Systems Engineering, pp. 13-17. [http://ceurws.org/Vol-1612/paper11.pdf] 
Huang, C. Y. and Wu, C. H. (2016). "Design and Implement an Interoperable Internet of Things Application Based on an Extended Ogc Sensorthings Api Standard". ISPRS - International Archives of the Photogrammetry, Remote Sensing and Spatial Information Sciences, pp. 263-266. [GS Search]

Hur, K., Chun, S., Jin, X., and Lee, K. H. (2015). "Towards a Semantic Model for Automated Deployment of IoT Services across Platforms". Proceedings - IEEE World Congress on Services, 2015. [GS Search]

Jara, Antonio J. e Olivieri, A. C., Bocchi, Y., Jung, M., Kastner, W., and Skarmeta, A. F. (2014). "Semantic Web of Things: an analysis of the application semantics for the IoT moving towards the IoT convergence". International Jounal of Web and Grid Services, pp. 244-272. [GS Search]

Jia, B., Li, W., Zhou, T. (2017). “A Centralized Service Discovery Algorithm via MultiStage Semantic Service Matching in Internet of Things". IEEE 19th International Conference on Computer Supported Cooperative Work in Design (CSCWD), pp. 422-427. [GS Search]

Kabisch, Sebastian e Anicic, D. (2016). "Thing Description as Enabler of Semantic Interoperability on the Web of Things". IoT Semantic Interoperability Workshop 2016. [https://www.iab.org/wp-content/IAB-uploads/2016/03/Thing-Description-asEnabler-of-Semantic-Interoperability-on-the-Web-of-Things.pdf]

Khriyenko, O., Terziyan, V., Kaikova, O. (2012). "User-assisted Semantic Interoperability in Internet of Things Visually-facilitated Ontology Alignment through Visually enriched Ontology and Thing Descriptions". 6th International Conference on Mobile Ubiquitous Computing, Systems, Services and Technologies. [Research Gate]

Kitchenham, B. and Charters, S. (2007). "Guidelines for performing systematic literature reviews in software engineering". Technical report. [https://userpages.unikoblenz.de/ laemmel/esecourse/slides/slr.pdf]

Kotis, K., Katasonov, A., Leino, J. (2012). "Aligning smart and control entities in the IoT”. Lecture Notes in Computer Science, pp. 39-50. [Research Gate]

Liu, M., Xu, Y., Hu, H., and Mohammed, A. W. (2016). "Semantic Agent-Based service middleware and simulation for smart cities". Sensors, pp. 1-25. [Research Gate]

Manate, Bogdan e Munteanu, V. I. Fortis, T. F. (2014). "Towards a smarter internet of things: Semantic visions". Proceedings - 2014 8th International Conference on Complex, Intelligent and Software Intensive Systems, CISIS 2014, pp. 582-587. [Research Gate]

Mathew, Sujith Samuel e Atif, Y., Sheng, Q. Z., and Maamar, Z. (2011). "Web of things: Description, discovery and integration". Proceedings - 2011 IEEE International Conferences on Internet of Things and Cyber, Physical and Social Computing, iThings/CPSCom 2011, pp. 9-15. [GS Search]

Mayer, S. and Zurich, E. T. H. (2013). "Service Integration in the Internet of Things. Computer Science", Computer Engineering, and Social Media (CSCESM), 2015. 
Mazayev, A., Martins, J. A., and Correia, N. (2017). Semantic web thing architecture. pages 43-46. 4th Experiment@ International Conference. [GS Search]

Mingozzi, E., Tanganelli, G., Vallati, C., Martinez, B., Mendia, I., GonzalezRodriguez, M. (2016). "Semantic-based context modeling for quality of service support in IoT platforms". IEEE 17th International Symposium on A World of Wireless, Mobile and Multimedia Networks (WoWMoM). [GS Search]

Pignotti, E., Beran, S., and Edwards, P. (2014). "What Does This Device Do?" Proceedings of 1st International Conference on IoT in Urban Space, pp. 56-61. [http://aura.abdn.ac.uk/bitstream/handle/2164/6078/pignotti_urbiot_14.pdf?sequence $=1 \&$ isAllowed $=\mathrm{y}]$

Pignotti, E. and Edwards, P. (2013). "Trusted tiny things: making the internet of things more transparent to users". Proceedings of the International Workshop on Adaptive Security. [GS Search]

Sahlmann, K. and Schwotzer, T. (2015). "MOCAP : Towards the SemanticWeb of Things". Semantics 2015. [Research Gate]

Schachinger, D., Fernbach, A., Kastner, W., Dinge, D. (2017). "Modeling framework for IoT integration of building automation systems". pp. 630-640. [GS Search]

Su, X., Zhang, H., Riekki, J., Ker”anen, A., Nurminen, J. K., Du, L. (2014). "Connecting IoT sensors to knowledge-based systems by transforming SenML to RDF”. Procedia Computer Science, pp. 215-222. [GS Search]

Sulistyo, S., Prinz, A. (2011). "PMG-Pro: A Model-Driven Development Method of Service-Based Applications". 15th International SDL Forum, pp. 138-153. [GS Search]

Suri, K., Gaaloul, W., Cuccuru, A., and Gerard, S. (2017). "Semantic Framework for Internet of Things-Aware Business Process Development”. pp. 214-219. [GS Search]

Thoma, M., Antonescu, A. F., Mintsi, T., Braun, T. (2013). "Linked services for M2M communication with Enterprise IT systems". 9th International Wireless Communications and Mobile Computing Conference, IWCMC 2013. [GS Search]

W3C (2015). "Thing description", [https://www.w3.org/WoT/IG/wiki/Thing_Description], outubro-2016.

Wang, W., De, S., Cassar, G., and Moessner, K. (2013). "Knowledge Representation in the Internet of Things : Semantic Modelling and its Applications". Journal for Control, Measurement, Electronics, Computing and Communications. [GS Search]

Yachir, A., Djamaa, B., Mecheti, A., Amirat, Y., and Aissani, M. (2016). "A Comprehensive Semantic Model for Smart Object Description and Request Resolution in the Internet of Things". Procedia Computer Science, pp. 147-154.

Yang, K., Li, S., Zhang, L., and Pan, G. (2010). "Semantic device bus for Internet of things". Proceedings - IEEE/IFIP International Conference on Embedded and Ubiquitous Computing, EUC 2010. [GS Search]

Yongan, Guo; Hongbo, Zhu; Longxiang, Y. (2016). "Service-Oriented Network Virtualization Architecture for Internet of Things". China Communications. [GS Search] 
Zhou, S., Liu, N., Li, G.-y., and Shi, Y.-m. (2015). "A Method for Service Dynamic Discovery Based on SOA4All Architecture in Semantic Web of Things". 8th International Symposium on Computational Intelligence and Design. [GS Search] 


\section{Apêndices}

Tabela 3 : Tabela completa dos trabalhos selecionados

\begin{tabular}{|c|c|c|c|}
\hline Título & Autores & Ano & Journal \\
\hline $\begin{array}{l}\text { A Centralized Service DiscoveryAlgorithm Via Multi-stage Semantic } \\
\text { Service Matching in Internet of Things }\end{array}$ & Jia, B. and Li, W. and Zhou, T. & 2017 & $\begin{array}{l}\text { IEEE International Conference on Computational Science } \\
\text { and Engineering and International Conference on } \\
\text { Embedded and Ubiquitous Computing. }\end{array}$ \\
\hline $\begin{array}{l}\text { A Comprehensive Semantic Model for Smart Object Description and } \\
\text { Request Resolution in the Internet of Things }\end{array}$ & $\begin{array}{l}\text { Huertas Celdran, A.a and Garcia Clemente, F.J.b } \\
\text { and Gil Perez, M.a and Martinez Perez, G.a }\end{array}$ & 2016 & Procedia Computer Science \\
\hline A flexible extension of WSDL to describe non-functional attributes & $\begin{array}{l}\text { Mingozzi, E.a and Tanganelli, G.a and Vallati, C.a } \\
\text { and Martinez, B.b and Mendia, I.b and Gonzalez- } \\
\text { Rodriquez. M. }\end{array}$ & 2016 & $\begin{array}{l}\text { International Conference On E-Business And Information } \\
\text { System Security }\end{array}$ \\
\hline $\begin{array}{l}\text { A Method for Service Dynamic Discovery Based on SOA4All } \\
\text { Architecture in Semantic Web of Things }\end{array}$ & Datta, S.K. and Bonnet, C. & 2016 & $\begin{array}{l}\text { International Symposium On Computational Intelligence } \\
\text { And Design }\end{array}$ \\
\hline A new service selection algorithm in USPIOT & Zhou, S. and Liu, N. and Li, G.-Y. and Shi, Y.-M. & 2016 & $\begin{array}{l}\text { leee International Conference On Computer Science And } \\
\text { Automation Engineering }\end{array}$ \\
\hline A scalable loT service search based on clustering and aggregation & $\begin{array}{l}\text { Yachir, A.a b and Djamaa, B.a and Mecheti, A.a } \\
\text { and Amirat, Y.b and Aissani, M.a }\end{array}$ & 2016 & $\begin{array}{l}\text { leee Internet Of Things And leee Cyber, Physical And } \\
\text { Social Computing }\end{array}$ \\
\hline A semantic enhanced sevvice proxy framework for internet of things & $\begin{array}{l}\text { Hirmer, P.a and Wieland, M.a and } \\
\text { BreitenbÃ } 1 / 4 c h e r, \text { U.b and Mitschang, B.a }\end{array}$ & 2016 & $\begin{array}{l}\text { International Conference On Green Computing And } \\
\text { Communications }\end{array}$ \\
\hline $\begin{array}{l}\text { A semantic processing framework for loT-enabled communication } \\
\text { systems }\end{array}$ & Huang, C.Y. and Wu, C.H. & 2016 & Lecture Notes In Computer Science \\
\hline $\begin{array}{l}\text { AFT: Adaptive Fibonacci-based Tuning Protocol for Service and } \\
\text { Resource discovery in the Internet of Things }\end{array}$ & Albalas, F. and Mardini, W. and Al-Soud, M. & 2017 & $\begin{array}{l}\text { 2nd International Conference on Fog and Mobile Edge } \\
\text { Computing, FMEC } 2017\end{array}$ \\
\hline Aligning smart and control entities in the loT & Wan, K.a and Alagar, V.b & 2015 & Lecture Notes In Computer Science \\
\hline An Ontology Design Pattern for loT Device Tagging Systems & Cho, G. and Chun, S. and Jin, X. and Lee, K. H. & 2015 & International Conference On The Internet Of Things(lot) \\
\hline Automated sensor registration, binding and sensor data provisioning & $\begin{array}{l}\text { Hirmer, P.a and Wieland, M.a and Breitenbacher, } \\
\text { U.b and Mitschang, B.a }\end{array}$ & 2015 & Ceur Workshop Proceedings \\
\hline $\begin{array}{l}\text { Connecting loT sensors to knowledge-based systems by transforming } \\
\text { SenML to RDF }\end{array}$ & $\begin{array}{l}\text { Han, S.N.a and Lee, G.M.b and Crespi, N.a and } \\
\text { Van Luong, N.c and Heo, K.d and Brut, M.e and } \\
\text { Gatellier, P.e }\end{array}$ & 2015 & Procedia Computer Science \\
\hline $\begin{array}{l}\text { Describing things in the Internet of Things: From CoRE link format to } \\
\text { semantic based descriptions }\end{array}$ & Sahlmann, K. and Schwotzer, T. & 2015 & $\begin{array}{l}\text { leee International Conference On Consumer Electronics- } \\
\text { Taiwan }\end{array}$ \\
\hline $\begin{array}{l}\text { Design and implement an interoperable Internet of Things application } \\
\text { based on an extended OGC sensorthings API Standard }\end{array}$ & $\begin{array}{l}\text { Su, X.a and Zhang, H.b and Riekki, J.a and } \\
\text { KerẪanen, A.c and Nurminen, J.K.d and Du, L.b }\end{array}$ & 2014 & $\begin{array}{l}\text { International Archives Of The Photogrammetry, Remote } \\
\text { Sensing And Spatial Information Sciences }\end{array}$ \\
\hline $\begin{array}{l}\text { Distributed Semantic Discovery for Web-of-Things Enabled Smart } \\
\text { Buildings }\end{array}$ & $\begin{array}{l}\text { Fredj, S.B.a and Boussard, M.a and Kofman, D.b } \\
\text { and Noirie, L.a }\end{array}$ & 2013 & $\begin{array}{l}\text { International Conference On New Technologies, Mobility } \\
\text { Andsecurity (Ntms) }\end{array}$ \\
\hline DPWSim: A devices profile for web services (DPWS) Simulator & Gao, F. and Derguech, W. & 2013 & leee Internet Of Things Journal \\
\hline $\begin{array}{l}\text { Enhancing CoAP proxy for semantic composition and multicast } \\
\text { communication }\end{array}$ & Pignotti, E. and Edwards, P. & 2013 & $\begin{array}{l}\text { Acm International Joint Conference On Pervasive And } \\
\text { Ubiquitous Computing And The Proceedings }\end{array}$ \\
\hline Improving Energy Efficiency in loT with Re-configurable Virtual Objects & Christophe, B. & 2012 & leee World Forum On Internet Of Things (Wf-lot) \\
\hline $\begin{array}{l}\text { Knowledge Representation in the Internet of Things: Semantic } \\
\text { Modellingand its Applications }\end{array}$ & Khriyenko, O. and Terziyan, V. and Kaikova, $\mathrm{O}$. & 2012 & Automatika \\
\hline Linked Services for M2M communication with Enterprise IT systems & $Y$. and $\mathrm{Hu}, \mathrm{H}$. & 2012 & $\begin{array}{l}\text { International Wireless Communications And Mobile } \\
\text { Computingconference (lwcmc) }\end{array}$ \\
\hline $\begin{array}{l}\text { Managing massive data of the internet of things through cooperative } \\
\text { semantic nodes }\end{array}$ & Kotis, K. and Katasonov, A. and Leino, J. & 2012 & leee International Conference On Semantic Computing \\
\hline MOCAP: Towards the semantic web of things & Christophe, B. & 2011 & Ceur Workshop Proceedings \\
\hline Modeling framework for loT integration of building automation systems & $\begin{array}{l}\text { Schachinger, Daniel and Fernbach, Andreas and } \\
\text { Kastner, Wolfgang }\end{array}$ & 2017 & $\begin{array}{l}\text { Modellierungsframework zur Integration von } \\
\text { Gebäudeautomationssystemen in das Internet der Dinge }\end{array}$ \\
\hline Multi-Agent recommendation system in internet of things & Forestiero, A. & 2017 & $\begin{array}{l}\text { 17th IEEE/ACM International Symposium on Cluster, } \\
\text { Cloud and Grid Computing, CCGRID } 2017\end{array}$ \\
\hline $\begin{array}{l}\text { Pevasive device and service discovery protocol in XBee sensor } \\
\text { network }\end{array}$ & $\begin{array}{l}\text { Akbar, S.R. and Kurniawan, W. and Ichsan, M.H.H. } \\
\text { and Arwani, I. and Handono, M.T. }\end{array}$ & 2017 & $\begin{array}{l}\text { International Conference on Advanced Computer Science } \\
\text { and Information Systems, ICACSIS } 2016\end{array}$ \\
\hline $\begin{array}{l}\text { PMG-Pro: A model-driven development method of service-based } \\
\text { applications }\end{array}$ & Sul & 2011 & Lecture Notes In Computer Science \\
\hline $\begin{array}{l}\text { SeCoMan: A Semantic-Aware Policy Framework for Developing } \\
\text { Privacy-Preserving and Context-Aware Smart Applications }\end{array}$ & $\begin{array}{l}\text { De, S.a and Barnaghi, P.a and Bauer, M.b and } \\
\text { Meissner, S.a }\end{array}$ & 2011 & leee Systems Journal \\
\hline $\begin{array}{l}\text { Semantic Agent-Based Service Middleware and Simulation for Smart } \\
\text { Cities }\end{array}$ & $\begin{array}{l}\text { Liu, Ming and } \mathrm{Xu}, \text { Yang and } \mathrm{Hu}, \text { Haixiao and } \\
\text { Mohammed, Abdul-Wahid }\end{array}$ & 2016 & Sensors Journal \\
\hline $\begin{array}{l}\text { Semantic Description, Discovery and Integration for the Internet } \\
\text { ofThings }\end{array}$ & $\begin{array}{l}\text { Mathew, S.S.a and Atif, Y.b and Sheng, Q.Z.a and } \\
\text { Maamar, Z.c }\end{array}$ & 2011 & $\begin{array}{l}\text { leee International Conference On Semantic Computing } \\
\text { (lcsc) }\end{array}$ \\
\hline Semantic device bus for Internet of things & Alam, S. and Noll, J. & 2010 & $\begin{array}{l}\text { International Conference On Embedded And Ubiquitous } \\
\text { Computing }\end{array}$ \\
\hline $\begin{array}{l}\text { Semantic framework for internet of things-aware business process } \\
\text { development }\end{array}$ & $\begin{array}{l}\text { Suri, K. and Gaaloul, W. and Cuccuru, A. and } \\
\text { Gerard S. }\end{array}$ & 2017 & $\begin{array}{l}\text { IEEE 26th International Conference on Enabling } \\
\text { Technologies: Infrastructure for Collaborative Enterprises, } \\
\text { WETICE } 2017\end{array}$ \\
\hline Semantic profiles to model the "Web of Things" & Yang, K. and Li, S. and Zhang, L. and Pan, G. & 2010 & $\begin{array}{l}\text { International Conference On Semantics, Knowledge, And } \\
\text { Grids }\end{array}$ \\
\hline Semantic web thing architecture & Mazayev, A. and Martins, J.A. and Correia, N. & 2017 & $\begin{array}{l}\text { 4th Experiment at International Conference: Online } \\
\text { Experimentation, exp.at } 2017\end{array}$ \\
\hline $\begin{array}{l}\text { Semantic-based context modeling for quality of service support in loT } \\
\text { platforms }\end{array}$ & Dai, C. and Wang, Z. & 2010 & $\begin{array}{l}\text { International Symposium On A World Of Wireless, Mobile } \\
\text { And Multimedia Networks }\end{array}$ \\
\hline SEMbySEM: A framework for sensors management & $\begin{array}{l}\text { Brunner, J.-S. and Goudou, J.F. and Gatellier, P. } \\
\text { and Beck, J. and Laporte, C.-E. }\end{array}$ & 2009 & CEUR Workshop \\
\hline Service integration in the Internet of Things & Guo, Yongan and Zhu, Hongbo and Yang, Longxiang & 2016 & $\begin{array}{l}\text { International Conference On Computer Science, Computer } \\
\text { Engineering, And Social Media }\end{array}$ \\
\hline Service modell & $\begin{array}{l}\text { Ehun, Sejin and Seo, Seungmin and Oh, Byungkook } \\
\text { and Lee, Kyong-Ho }\end{array}$ & 2015 & $\begin{array}{l}\text { Federated Conference On Computer Science And } \\
\text { Information Systems }\end{array}$ \\
\hline $\begin{array}{l}\text { Service-Oriented Network Virtualization Architecture for Internet } \\
\text { offhings }\end{array}$ & $\begin{array}{l}\text { Hur, Kangho and Chun, Sejin and Jin, Xiongnan and } \\
\text { Lee, Kyong-Ho }\end{array}$ & 2015 & China Communications \\
\hline $\begin{array}{l}\text { Towards A Semantic Model for Automated Deployment of loT Services } \\
\text { AcrossPlatforms }\end{array}$ & $\begin{array}{l}\text { Charpenay, Victor and Kabisch, Sebastian and } \\
\text { Anicic, Darko and Kosch, Harald }\end{array}$ & 2015 & leee World Congress On Services \\
\hline $\begin{array}{l}\text { Trusted tiny things: Making the Internet of Things more transparent to } \\
\text { users }\end{array}$ & bert, Jean & 2014 & International Workshop On Adaptive Security \\
\hline Ubiquitous sevice capability modeling and similarity based searching & $\begin{array}{l}\text { Etelapera, Matti and Vecchio, Massimo and } \\
\text { Giaffreda, Raffaele }\end{array}$ & 2014 & Lecture Notes In Computer Science \\
\hline $\begin{array}{l}\text { User-assisted semantic interoperability in internet of things visually- } \\
\text { facilitated ontology alignment through visually-enriched ontology and } \\
\text { thing descriptions }\end{array}$ & $\begin{array}{l}\text { Ara, Safina Showkat and Shamszaman, Zia Ush and } \\
\text { Chong, llyoung }\end{array}$ & 2014 & $\begin{array}{l}\text { International Conference On Mobile Ubiquitous Computing, } \\
\text { Systems, Services And Technologies }\end{array}$ \\
\hline Web of things: Descriptio & $\begin{array}{l}\text { Wang, Wei and De, Suparna and Cassar, Gilbert } \\
\text { and Moessner, Klaus }\end{array}$ & 2013 & $\begin{array}{l}\text { International Conferences On Internet Of Things And } \\
\text { Cyber, Physical And Social Computing, Ithings }\end{array}$ \\
\hline $\begin{array}{l}\text { Web-of-Objects Based User-Centric Semantic Service } \\
\text { CompositionMethodology in the Internet of Things }\end{array}$ & $\begin{array}{l}\text { Thoma, Matthias and Antonescu, Alexandru-Florian } \\
\text { and Mintsi, Theano andBraun, Torsten }\end{array}$ & 2013 & International Journal Of Distributed Sensor Networks \\
\hline What Does This Device Do? & $\begin{array}{l}\text { Pignotti, Edoardo and Beran, Stanislav and } \\
\text { Edwards, Peter }\end{array}$ & 2014 & Conference On lot In Urban Space \\
\hline
\end{tabular}

